\title{
Repetitive Transcranial Magnetic Stimulation to Treat Depression and Insomnia with Chronic Low Back Pain
}

Seoul ChukChuk Pain Hospital, *Department of Anesthesiology and Pain Medicine, Soonchunhyang Seoul Hospital, College of Medicine, Soonchunhyang University,

${ }^{\dagger}$ Godoil Spine and Pain Hospital, Seoul, Korea

Eun Jung Park, Se Jin Lee*, Do Yle Koh ${ }^{\dagger}$, and Yoo Mi Han*

Transcranial magnetic stimulation (TMS) is a noninvasive and safe technique for motor cortex stimulation. TMS is used to treat neurological and psychiatric disorders, including mood and movement disorders. TMS can also treat several types of chronic neuropathic pain. The pain relief mechanism of cortical stimulation is caused by modifications in neuronal excitability. Depression is a common co-morbidity with chronic pain. Pain and depression should be treated concurrently to achieve a positive outcome. Insomnia also frequently occurs with chronic lower back pain. Several studies have proposed hypotheses for TMS pain management. Herein, we report two cases with positive results for the treatment of depression and insomnia with chronic low back pain by TMS. (Korean J Pain 2014; 27: 285-289)

\section{Key Words:}

back pain, chronic pain, depression, insomnia, transcranial magnetic stimulation.

Low back pain is a very common health problem, and the lifetime prevalence of low back pain is estimated at $60 \%$ to $70 \%$. It is often difficult to treat.

Chronic spinal pain, such as lower back pain, has been associated with sleep disturbances, including reduced sleep duration and quality [1].

Depression is a co-morbid disease in patients with chronic back pain [2]. Insomnia and depression are detrimental clinical symptoms in chronic low back pain patients.

Improvement of insomnia and depression in patients with chronic low back pain improves the overall prognosis.

Transcranial magnetic stimulation (TMS) is a non- invasive technique that stimulates the brain cortex. It uses a magnetic field to generate weak electric currents in the cortex. In one study, the brain can be stimulated using external magnetic stimulation with TMS [3]. TMS produces brief magnetic pulses, which pass easily and painlessly through the skull and into the brain. TMS is commonly used to treat neurological and psychiatric disorders including mood disorders, dystonia, and Parkinson's disease [4]. TMS has been used for pain relief in chronic pain syndromes such as fibromyalgia, complex regional pain syndrome, and other neuropathic pain conditions [5]. This report presents two cases of chronic low back pain with con-

Received October 18, 2013. Revised May 9, 2014. Accepted May 12, 2014.

Correspondence to: Se Jin Lee

Department of Anesthesiology and Pain Medicine, Soonchunhyang University Seoul Hospital, 59 Daesagwan-gil, Yongsan-gu, Seoul 140-743, Korea

Tel: +82-2-709-9302, Fax: +82-2-790-0394, E-mail: alvesta@schmc.ac.kr

(a) This is an open-access article distributed under the terms of the Creative Commons Attribution Non-Commercial License (http:// creativecommons.org/licenses/by-nc/3.0/), which permits unrestricted non-commercial use, distribution, and reproduction in any medium, provided the original work is properly cited.

Copyright (c) The Korean Pain Society, 2014 
current insomnia and depression. We performed TMS and obtained positive results.

\section{CASE REPORT}

\section{Patient 1}

A 65-year-old woman had a 5-year history of lower back pain following posterolateral interbody fusion surgery. She was treated with fluoroscopically guided epidural injections and medications including non-steroidal anti-inflammatory drugs and muscle relaxants for 6 months. Conventional treatment yielded $20-30 \%$ pain improvement for 2-3 days. After that period, pain returned to pretreatment levels. The patient complained of sleep disturbances and was depressed from experiencing severe pain and activity restriction. We performed TMS with the TAMAS TMS Therapy system (CR Technology Inc., Daejeon, Korea) (Fig. 1). The TMS machine was set at a frequency of $1 \mathrm{~Hz}$ and $100 \%$ of the resting motor threshold for $20 \mathrm{~min}$. This delivered 1200 pulses per session, five times per week for 4 weeks. A figure-of-eight-shaped coil magnetic stimulator was placed on the skull overlying the left prefrontal cortex. We assessed treatment outcomes using the Pain Numerical Rating Scale (NRS), the Beck Depression Inventory (BDI), the Insomnia Severity Index (ISI) and the Pain Disability Index (PDI). The patient filled out questionnaires prior to and following treatment (Fig. 2). NRS ( $0=$ no pain, 100 $=$ worst pain imaginable) decreased from 80 to 30 , and depression, assessed by the BDI self-report scale (0 to 63), decreased from 50 (extreme depression) to 11 (mild mood disturbance). Insomnia, assessed by ISI (0 to 28), improved from 23 (severe insomnia) to 10 (sub-threshold insomnia). Disability was assessed by PDI (0 to 70), and also improved from 63 to 32 . The patient was very satisfied with the treatment results, and symptom improvement was maintained for at least 3 months following treatment. Any side effects such as headache and hearing problem were not found.

\section{Patient 2}

A 61-year-old female patient had a 1-year history of lower back and bilateral leg pain. She had a laminectomy 1 year previously, and the pain was aggravated following the operation. The patient suffered from severe pain, sleep problems, and depression. She was treated with medications including non-steroidal anti-inflammatory drugs and muscle relaxants for 3 months. Caudal epidural block and transforaminal epidural block were performed without pain improvement. TMS was performed with the aforementioned stimulation settings. The patient received a total of 15 sessions, five times per week for 3 weeks. The questionnaires were given before and after treatment (Fig. 2). Pain improved by 50\% compared to baseline levels as assessed by NRS decreasing from 80 to 40. Insomnia improved from moderate levels to sub-threshold levels as assessed by ISI (0 to 28), improving from 21 to 13. Depression as assessed by BDI decreased from 26 (moderate) to 13 (mild mood disturbance). Activity also improved from 55 to 27. The patient was very satisfied with the results, and the effects were maintained for at least 3 months following conclusion of the treatment. Any side effects such as headache and hearing problem were not found.
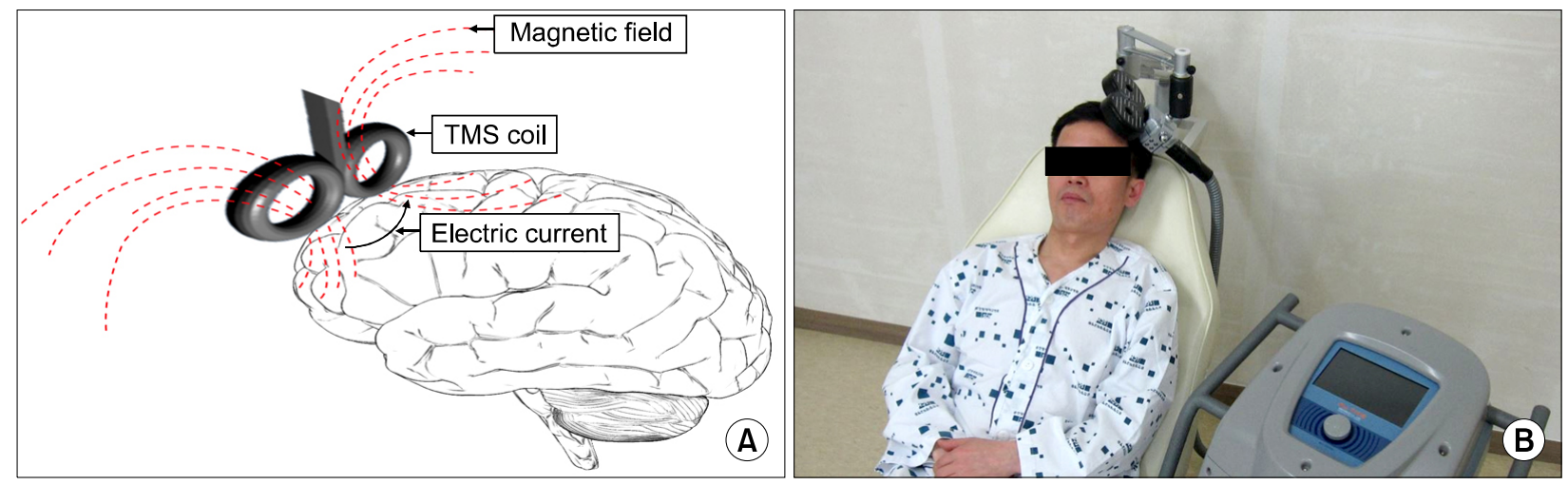

Fig. 1. (A) Transcranial magnetic stimulation machine delivers the electric currents to the brain through the magnetic stimulator coil. (B) A figure-of-eight-shaped coil magnetic stimulator is placed on the skull overlying the left prefrontal cortex. 
A Change in pain based on the NRS

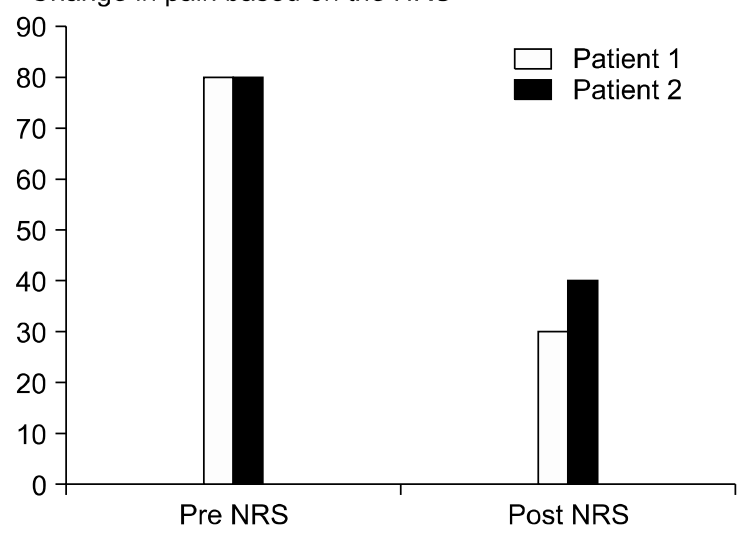

C Change in sleep disturbance based on the ISI

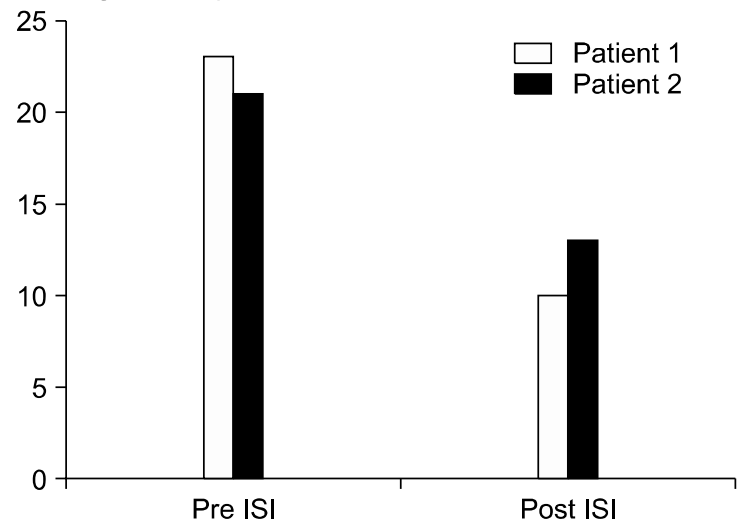

B Change in depression based on the BDI

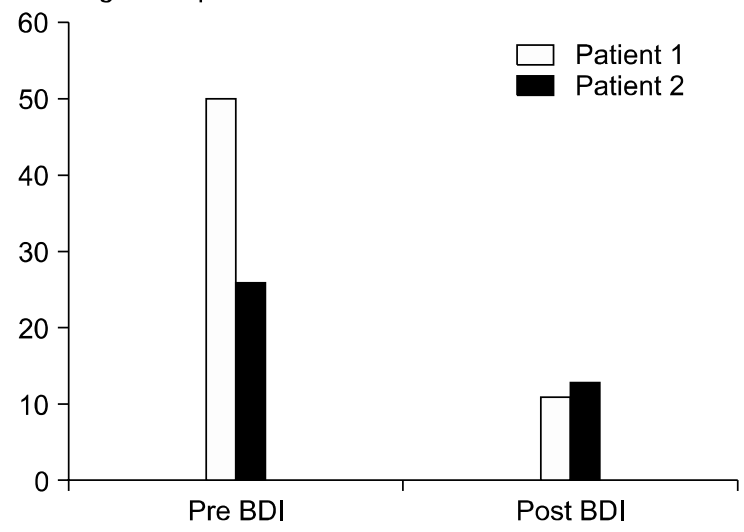

D Change in daily activities based on the PDI

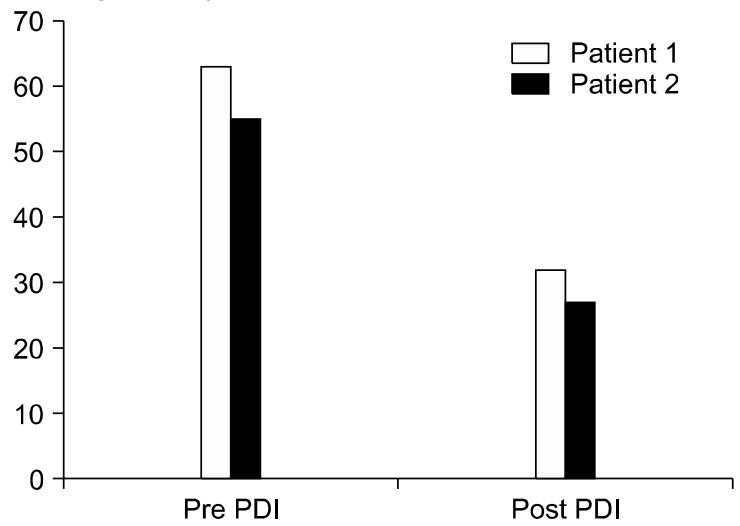

Fig. 2. (A) Change in NRS after the TMS. (B) Change in BDI after the TMS. (C) Change in ISI after the TMS. (D) Change in PDI after the TMS. BDI: Beck depression inventory (0-63), ISI: insomnia severity index (0-28), NRS: numerical rating scale $(0-100)$, PDI: pain disability index $(0-70)$, TMS: transcranial magnetic stimulation.

\section{DISCUSSION}

Various neurostimulation methods have been applied in the treatment of chronic pain including the following: peripheral nerve stimulation (PNS), spinal cord stimulation (SCS), deep brain stimulation (DBS), motor cortex stimulation (MCS), and repetitive transcranial magnetic stimulation (rTMS) [6]. MCS and rTMS are emerging cortical stimulation techniques to treat patients with chronic pain. The mechanism of cortical stimulation for pain relief is based on the modification of neuronal excitability. In chronic pain states, pain conduction systems are injured resulting in chronic pain and a lowered pain threshold. Cerebral cortex stimulation modulates abnormal thalamic activity which alleviates pain in these syndromes [7].

Depression is a common co-morbidity in chronic pain. Several studies have reported the prevalence of major de- pression in chronic lower back pain over a 6-month period (21-45\%) [8]. Depression is the result of the imbalance and dysfunction of neurotransmitters such as serotonin, norepinephrine, and dopamine [9]. Several studies have shown that pain and depression are conducted through the same descending pathways in the central nervous system forming a biological link between these two conditions [2]. Severe pain, restriction of daily activities, and pain refractory to treatment are related to depression. Patients with both pain and depression should be treated for these conditions simultaneously for optimal treatment outcomes [2]. TMS has been used in the treatment of psychiatric disorders. A TMS machine delivers a magnetic field to the brain using a magnetic stimulator to stimulate neurons to release neurotransmitters into the synaptic cleft. These neurotransmitters affect mood, cortical excitability, and other brain activities [10]. There is a study that TMS treat- 
ment of the left prefrontal cortex for at least 3 weeks has an antidepressant effect [11]. George et al. [12] showed remission in $30-40 \%$ of cases which was maintained for 3 months following treatment in $58 \%$ of the patients. We assessed clinical improvement of depression using the BDI. The BDI is a useful questionnaire utilizing a self-rated scale for observing the level of depression. A reduction in BDI scores was achieved in both patients following the conclusion of the treatment. The depression grade decreased from moderate and extreme depression to a mild mood disturbance. Mood improvement was maintained at the 3-month follow up.

Insomnia is a common co-morbid condition with chronic lower back pain. Insomnia is related with reduced quality of life. Sleep impairment occurred in 55\% of chronic lower back pain patients, and over $50 \%$ of insomniacs had moderate to severe levels of insomnia [13]. The severity of insomnia was measured by the Insomnia Severity Index (ISI). This questionnaire is a valid and reliable tool that contains seven statements relating to insomnia severity, producing a range from 0 to 28. A higher score corresponds to a greater severity. In our report, insomnia reduced from moderate and severe levels to sub-threshold insomnia. The quality of sleep was better after treatment. A TMS study evaluating insomnia showed a significant improvement in sleep problems, with a lower recurrence rate compared to treatment with medication and psychotherapy [14].

TMS has been used for the treatment of various chronic neuropathic pain disorders, including phantom limb pain, fibromyalgia, complex regional pain syndrome, and central pain following stroke or spinal cord injury [5]. The mechanism of TMS therapy has not been previously addressed. TMS acts on pain-modulating systems in the diencephalon and descending pain pathways from the brainstem to the spinal cord [15]. Several studies proposed hypotheses for the mechanism of chronic pain management with TMS. GABAergic inhibitory neurotransmission may play a role in pain modulation with TMS [16]. Endogenous opioid systems may also be affected by TMS-induced analgesia depending on the stimulation site [17]. TMS has produced analgesia with chronic pain, but the improvement depends on various factors, including the stimulation frequency, stimulation site, and treatment duration [18]. Treatment duration is important to maintain the treatment effects. TMS provided on consecutive days yielded long-lasting effects.

Current published clinical trials on the efficacy of rTMS in treating depression are almost certainly suboptimal (e.g. duration of two weeks). The data support positive outcomes for rTMS, but not large clinical effects [18]. However, a few studies suggest that a longer course of rTMS is necessary for optimal therapeutic outcomes. Bretlau et al. [19] found that over 3 weeks, the active rTMS treatment was superior to the sham TMS. In our study, subjects with depression, insomnia and chronic back pain were treated each weekday for 3 to 4 weeks.

Stimulation frequency relates to synaptic changes. Generally higher frequencies $(>5 \mathrm{~Hz}$ ) are excitatory, and lower frequencies $(<1 \mathrm{~Hz})$ are inhibitory. Some studies suggest TMS at $10 \mathrm{~Hz}$ had better results than $5 \mathrm{~Hz}$, and $1 \mathrm{~Hz}$ did not produce a significant effect [15]. However, our patients received TMS at a $1-\mathrm{Hz}$ frequency, and experienced significant pain relief. TMS effects vary amongst individuals, and further study is required to determine the optimal treatment protocols. We used the Pain Disability Index (PDI) because it provides a simple questionnaire to assess the impact of pain in terms of activity restriction. The questionnaire addresses seven items on a scale of 0-70: family and home responsibilities, recreation, social activity, occupation, sexual behavior, self-care, and lifesupport activity. A higher score corresponds to greater disability due to pain. We observed improvement in daily function following TMS therapy in these cases.

TMS is a non-invasive and safe technique. These patients did not report any side effects or complications. Machii et al. [20] reviewed studies evaluating TMS published between January 1998 and December 2003. The most common adverse effect was a mild and instant headache, which occurred in $23 \%$ of patients. Serious adverse effects, including seizures, were rare. TMS is a safe treatment modality when manufacturer guidelines are followed, and patients are monitored during the treatment. These cases had positive clinical outcomes regarding pain, insomnia, and depression. Prospective randomized controlled studies are needed to confirm the efficacy of TMS treatment of insomnia, depression, and pain from chronic low back pain syndrome. Future studies should focus on TMS instrument settings, including the stimulation site, frequency, and duration. In addition, future investigations should define the mechanism of TMS.

In conclusion, these cases show the successful results from TMS therapy for the treatment of depression, sleep disorders, and pain. We suggest that rTMS could be a safe 
and effective treatment option for patients with chronic low back pain resulting in depression and insomnia. Further studies are required to verify the positive outcomes of TMS treatment for chronic low back pain.

\section{REFERENCES}

1. Kelly GA, Blake C, Power CK, O'keeffe D, Fullen BM. The association between chronic low back pain and sleep: a systematic review. Clin J Pain 2011; 27: 169-81.

2. Bair MJ, Robinson RL, Katon W, Kroenke K. Depression and pain comorbidity: a literature review. Arch Intern Med 2003; 163: 2433-45.

3. Barker AT, Jalinous R, Freeston IL. Non-invasive magnetic stimulation of human motor cortex. Lancet 1985; 1: 1106-7.

4. Simons W, Dierick M. Transcranial magnetic stimulation as a therapeutic tool in psychiatry. World J Biol Psychiatry 2005; 6: 6-25.

5. Leung A, Donohue M, Xu R, Lee R, Lefaucheur JP, Khedr EM, et al. rTMS for suppressing neuropathic pain: a meta-analysis. J Pain 2009; 10: 1205-16.

6. Rokyta R, Fricová J. Neurostimulation methods in the treatment of chronic pain. Physiol Res 2012; 61 Suppl 2: s23-31.

7. Zaghi S, Heine N, Fregni F. Brain stimulation for the treatment of pain: a review of costs, clinical effects, and mechanisms of treatment for three different central neuromodulatory approaches. J Pain Manag 2009; 2: 339-52.

8. Krishnan KR, France RD, Pelton S, McCann UD, Davidson J, Urban BJ. Chronic pain and depression. II. Symptoms of anxiety in chronic low back pain patients and their relationship to subtypes of depression. Pain 1985; 22: 289-94.

9. Maletic V, Robinson M, Oakes T, lyengar S, Ball SG, Russell J. Neurobiology of depression: an integrated view of key findings. Int J Clin Pract 2007; 61: 2030-40.

10. Paus T, Barrett J. Transcranial magnetic stimulation (TMS) of the human frontal cortex: implications for repetitive TMS treatment of depression. J Psychiatry Neurosci 2004; 29:
268-79.

11. George MS, Lisanby SH, Avery D, McDonald WM, Durkalski $V$, Pavlicova M, et al. Daily left prefrontal transcranial magnetic stimulation therapy for major depressive disorder: a sham-controlled randomized trial. Arch Gen Psychiatry 2010; 67: 507-16.

12. George MS, Taylor JJ, Short EB. The expanding evidence base for rTMS treatment of depression. Curr Opin Psychiatry 2013; 26: 13-8.

13. Tang NK, Wright KJ, Salkovskis PM. Prevalence and correlates of clinical insomnia co-occurring with chronic back pain. J Sleep Res 2007; 16: 85-95.

14. Jiang CG, Zhang T, Yue FG, Yi ML, Gao D. Efficacy of repetitive transcranial magnetic stimulation in the treatment of patients with chronic primary insomnia. Cell Biochem Biophys 2013; 67: 169-73.

15. Lefaucheur JP. Use of repetitive transcranial magnetic stimulation in pain relief. Expert Rev Neurother 2008; 8: 799-808.

16. Barr MS, Farzan F, Davis KD, Fitzgerald PB, Daskalakis ZJ. Measuring GABAergic inhibitory activity with TMS-EEG and its potential clinical application for chronic pain. J Neuroimmune Pharmacol 2013; 8: 535-46.

17. de Andrade DC, Mhalla A, Adam F, Texeira MJ, Bouhassira D. Neuropharmacological basis of rTMS-induced analgesia: the role of endogenous opioids. Pain 2011; 152: 320-6.

18. Loo CK, Mitchell PB. A review of the efficacy of transcranial magnetic stimulation (TMS) treatment for depression, and current and future strategies to optimize efficacy. J Affect Disord 2005; 88: 255-67.

19. Bretlau LG, Lunde M, Lindberg L, Undén M, Dissing S, Bech P. Repetitive transcranial magnetic stimulation (rTMS) in combination with escitalopram in patients with treatmentresistant major depression: a double-blind, randomised, sham-controlled trial. Pharmacopsychiatry 2008; 41: 41-7.

20. Machii K, Cohen D, Ramos-Estebanez C, Pascual-Leone A. Safety of rTMS to non-motor cortical areas in healthy participants and patients. Clin Neurophysiol 2006; 117: 455-71. 